Check for updates

Cite this: Phys. Chem. Chem. Phys., 2017, 19, 12776

Received 18th March 2017 Accepted 27th April 2017

DOI: $10.1039 / c 7 c p 01733 b$

rsc.li/pccp

\section{Isomerisation of an intramolecular hydrogen-bonded photoswitch: protonated azobis(2-imidazole) $\uparrow$}

\begin{abstract}
James N. Bull, (D) Michael S. Scholz, Neville J. A. Coughlan and Evan J. Bieske (D)*
Photoisomerisation of protonated azobis(2-imidazole), an intramolecular hydrogen-bonded azoheteroarene photoswitch molecule, is investigated in the gas phase using tandem ion mobility mass spectrometry. The $E$ and $Z$ isomers exhibit distinct spectral responses, with $E-Z$ photoisomerisation occurring over the $360-520 \mathrm{~nm}$ range (peak at $460 \mathrm{~nm}$ ), and $Z-E$ photoisomerisation taking place over the $320-420 \mathrm{~nm}$ range (peak at $390 \mathrm{~nm}$ ). A minor photodissociation channel involving loss of $\mathrm{N}_{2}$ is observed for the $E$-isomer with a maximum efficiency at $390 \mathrm{~nm}$, blue-shifted by $\approx 70 \mathrm{~nm}$ relative to the wavelength for maximum photoisomerisation response. Loss of $\mathrm{N}_{2}$ is also the predominant collision-induced dissociation channel. Electronic structure calculations suggest that $E$-isomer photoisomerisation involves $\mathrm{S}_{1}\left(\pi \pi^{\star}\right)$ excitation, whereas the $Z$-isomer photoisomerisation involves $S_{2}\left(\pi \pi^{\star}\right)$ excitation. Conversion between the $E$ and $Z$ isomers through collisional excitation, which is calculated to occur through both inversion and torsion pathways, is investigated experimentally by colliding the molecular ions with nitrogen buffer gas over a range of electric fields. This study demonstrates the versatility of tandem ion mobility mass spectrometry for exploring the isomerisation of molecular photoswitches initiated by either light or collisions.
\end{abstract}

\section{Introduction}

Molecules consisting of two arene or heteroarene moieties linked by an azo group $(-\mathrm{N}=\mathrm{N}-)$ exhibit reversible $E$ (trans) $\rightarrow Z$ (cis) photoisomerisation about the azo bond, and in some cases possess the desirable characteristics for a molecular photoswitch, including high isomerisation quantum yield, photostability, and possibility of reversible photoisomerisation using different colours of visible light. ${ }^{1-3}$ They have potential applications as biological probes, ${ }^{2,4}$ actuators in molecular machines, ${ }^{5-7}$ molecular units for data storage, ${ }^{8}$ photochromic ionic liquid solvents, ${ }^{9}$ linking units to control peptide conformation and enzyme activity, ${ }^{10-13}$ and in photopharmacology. ${ }^{14-16}$ In a recent development, Weston et $a .^{17}$ demonstrated that protonated azobis(2-imidazole) (PABI, Fig. 1), an imidazole-based photoswitch, can photoswitch an aqueous solution's $\mathrm{pH}$ by exploiting a $\mathrm{p} K_{\mathrm{a}}$ difference of $\approx 1.3$ between the $E$ and $Z$ isomers in their ground electronic states. The origin of the $E-Z$ isomer $\mathrm{p} K_{\mathrm{a}}$ difference is a stabilising hydrogen bond for the $Z$-isomer with the two imidazole rings

School of Chemistry, University of Melbourne, Parkville, VIC 3010, Australia. E-mail: evanjb@unimelb.edu.au

$\dagger$ Electronic supplementary information (ESI) available: Synthetic procedure; all PABI isomers and isomerisation barriers; review of the level of electronic structure theory; procedure for measuring collision cross-sections; ATDs recorded following irradiation of the photoswitch solution in the electrospray syringe with blue light. See DOI: 10.1039/c7cp01733b sharing the additional proton. Imidazole-based azo moieties such as PABI are increasingly being used in photoswitching applications due to higher photoisomerisation quantum yields and slower thermal reversion rates compared with conventional azoarene based photoswitches. ${ }^{1}$ Because of their growing importance, it is desirable to understand the photochemistry and photoswitching behaviour of azoheteroarenes in a range of environments.

Photoisomerisation of azoheteroarenes such as PABI is generally assumed to occur through similar mechanisms to azobenzene. ${ }^{18}$ The $\mathrm{S}_{1}\left(\mathrm{n} \pi^{*}\right) \leftarrow \mathrm{S}_{0}$ transition for $E$-azobenzene is symmetry forbidden due to the planar geometry, whereas the $\mathrm{S}_{2}\left(\pi \pi^{*}\right) \leftarrow \mathrm{S}_{0}$ transition has high oscillator strength $(\approx 0.8){ }^{3}$ Time-resolved measurements in the gas phase demonstrate that $E$-azobenzene molecules in the $\mathrm{S}_{2}$ state undergo internal conversion to the $S_{1}$ state on a 110-170 fs timescale, followed by

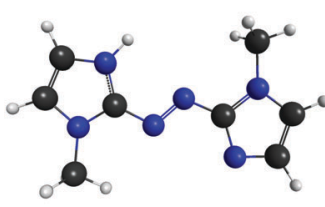

E1

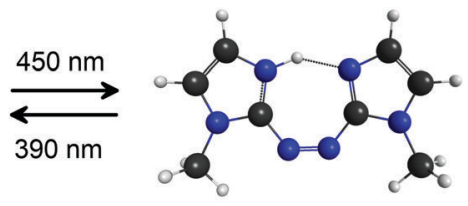

Z1
Fig. $1 E 1$ and $Z 1$ isomers of protonated azobis(2-imidazole), PABI. The Z1-isomer has a hydrogen bond between the imidazole rings and is calculated to lie $5 \mathrm{~kJ} \mathrm{~mol}^{-1}$ above the E1-isomer. 
isomerisation on $\mathrm{a} \approx 500$ fs timescale $(\approx 25 \%$ quantum yield $)$ and recovery of the $S_{0}$ state. ${ }^{19-21}$ The $S_{3}\left(\pi \pi^{*}\right)$ and $S_{4}\left(\pi \pi^{*}\right)$ states of $E$-azobenzene, which are associated with weak absorption profiles overlapping the blue end of the $S_{2}$ absorption band, undergo direct internal conversion to the $S_{0}$ state without isomerisation (although ground state isomerisation of the hot molecules may subsequently occur). ${ }^{19}$ Experiment and theory suggest that $\mathrm{S}_{1}$-mediated isomerisation occurs through both inversion and torsion of the azo bond, with the relative importance of the two mechanisms depending on the excitation wavelength, identity of the arene or heteroarene rings, functional group substitution, and solvent. ${ }^{19-25}$

In the current work we have investigated the isomerisation of $E$ and $Z$ isomers of PABI in the gas phase initiated through absorption of light or collisional excitation. The experimental approach involves tandem ion mobility spectrometry (IMS), whereby charged isomers drifting under the influence of an electric field through a buffer gas are separated according to their drift speeds, which depend on their collision cross sections. The $E$ and $Z$ isomers are separated in a first IMS stage and are then excited by either light or collisions, with separation of the resulting isomers in the second IMS stage. By monitoring the photoisomer yield as a function of wavelength, one can record a photoisomerisation action (PISA) spectrum that reflects the photoresponse of a particular isomer in the gas phase. A similar approach has been used by our group to investigate the photoisomerisation of polyene dye molecules, ${ }^{26,27}$ retinal protonated Schiff base, ${ }^{28-30}$ merocyanine,${ }^{31}$ and recently by others to investigate an alkene-pyridinium chromophore embedded in a crown ether. ${ }^{32}$ One of the crucial issues we wish to address for PABI is whether reversible photoswitching is possible using light of different wavelengths, a characteristic of an ideal molecular photoswitch. We also aim to provide structural and spectroscopic data for PABI in the gas phase that can be compared with predictions from high-level theoretical calculations for the structures and relative energies for the various isomers, and the energies and intensities of their electronic transitions.

\section{Experimental methods}

Azobis(2-imidazole) was synthesised following the procedure of Weston et al. ${ }^{17}$ (see $\mathrm{ESI} \dagger$ ). The ion mobility experiments were performed using a custom IMS-IMS-MS apparatus illustrated in Fig. $2 .^{33}$ Briefly, a solution of $\approx 10^{-4} \mathrm{~mol} \mathrm{~L}^{-1}$ analyte dissolved in methanol and adjusted to $\mathrm{pH} \approx 4$ with acetic acid was loaded into a syringe, which was connected via a section of silica capillary

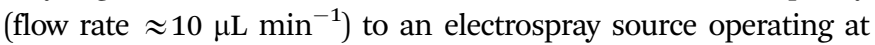
$\approx 3 \mathrm{kV}$. Electrosprayed ions were transferred via a heated capillary into a RF ion funnel (IF1), which gathered the ions and propelled them towards an electrostatic ion gate (IG1) that was opened to inject $\approx 100 \mu$ s packets of ions at $20 \mathrm{~Hz}$ into the first IMS region (IMS1). In IMS1 the ions were propelled by an electric field $\left(44 \mathrm{~V} \mathrm{~cm}^{-1}\right)$ through $\mathrm{N}_{2}$ buffer gas at a pressure of $\approx 6$ Torr. The $E$ and $Z$ isomers become spatially separated because of differences in their collision cross-sections. At the end of IMS1 a BradburyNielsen ion gate (IG2) was opened briefly $(100 \mu$ s) to select either

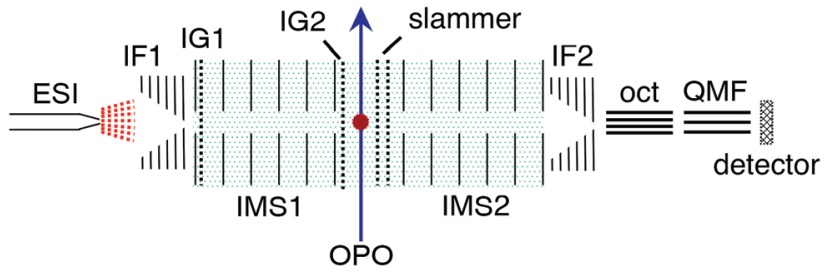

Fig. 2 Schematic illustration of the IMS-IMS-QMF instrument showing electrospray ionisation (ESI), ion funnels (IF1 and IF2), ion gates (IG1 and IG2), ion mobility drift regions (IMS1 and IMS2), light beam passing through the photoisomerisation zone (OPO); octupole ion guide (oct), and quadrupole mass filter (QMF). Total drift region length (IMS1 + IMS2) is $0.9 \mathrm{~m}$, $\mathrm{N}_{2}$ buffer gas pressure is $\approx 6$ Torr, and drift field is $44 \mathrm{~V} \mathrm{~cm}^{-1}$.

the $E$ or $Z$ isomer. These mobility-selected ions were excited immediately after IG2 with either a pulse of light or by collisional excitation in a short collision region with an adjustable electric field (slammer). The ions then passed through a second IMS region (IMS2) which serves to separate isomers formed by light or collisions. At the end of IMS2 a second ion funnel (IF2) collected the ions and introduced them into a differentially pumped octupole ion guide and then a quadrupole mass filter (QMF) that massselected the ions before they reached a channeltron ion detector. The detector was connected to a multichannel scaler that produced a histogram of ion counts against arrival time, corresponding to an arrival time distribution (ATD). The mobility resolution for singlycharged ions is typically 70-80. ${ }^{33}$ Collision cross-sections with $\mathrm{N}_{2}$ buffer gas were calibrated with respect to the cross sections for a series of tetraalkylammonium salts (see ESI $\dagger$ ).

For the photoisomerisation measurements, every second mobility-selected ion packet was irradiated using light from an optical parametric oscillator (OPO, EKSPLA NT342B, 2-3 mJ cm ${ }^{-2}$ pulse). The difference between the light-on and light-off ATDs (action signal) reflects the photoisomerisation response. The action signal was normalised with respect to total light-off ion signal and light pulse fluence.

For the collision-induced isomerisation measurements, the mobility-selected ion packets were energised in the slammer region between two electrodes that were separated by $3 \mathrm{~mm}$ and whose apertures were covered by $90 \%$ transmission nickel mesh. The slammer potential difference could be adjusted to increase the ions' drift velocity and energy of collisions with $\mathrm{N}_{2}$ buffer gas, promoting thermal isomerisation. ${ }^{34,35}$ In a collisioninduced isomerisation experiment, ATDs were measured as a function of slammer potential difference, $\Delta V$, typically ranging from 20 to $200 \mathrm{~V}\left(70-667 \mathrm{~V} \mathrm{~cm}^{-1}\right)$. Potentials of all upstream electrodes were appropriately adjusted such that electric fields in other IMS drift regions were unaffected.

\section{Theoretical methods}

Electronic structure calculations for all PABI isomers were performed using the PSI $4,{ }^{36}$ GAMESS-US, ${ }^{37}$ ORCA 3.0.3, ${ }^{38}$ and Gaussian 09 software packages. ${ }^{39}$ Ground state isomer energies were computed at either the CCSD(T)/cc-pVTZ or DLPNO-CCSD(T)/cc-pVTZ 
level of theory at the MP2//cc-pVTZ or $\omega \mathrm{B} 97 \mathrm{X}-\mathrm{D} / / \mathrm{cc}-\mathrm{pVTZ}$ geometry. ${ }^{40-44}$ The DLPNO-CCSD(T) method has the advantage

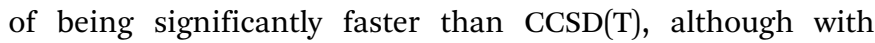
a sacrifice in accuracy of typically $\pm 4 \mathrm{~kJ} \mathrm{~mol}^{-1}$ relative to $\operatorname{CCSD}(\mathrm{T})$ - see further details in the ESI. $\dagger^{41}$ Vibrational zero-point energy corrections were determined from $\omega \mathrm{B} 97 \mathrm{X}-\mathrm{D} / / \mathrm{cc}-\mathrm{pVTZ}$ vibrational frequency calculations. The multistate XMCQDPT2/GEN and EOM-CCSD/GEN level of theory were used for determining excited state energies of the PABI isomers. ${ }^{45,46}$ The GEN basis set is the cc-pVTZ basis set from which $f$ functions are excluded for computational tractability. Several recent studies used the multistate XMCQDPT2 method to provide reliable excited state potential energy surfaces for understanding the photoisomerisation of stilbene and azobenzene. ${ }^{23,47}$ These studies provide a detailed account of the CASSCF reference state averaging needed for a physically meaningful wavefunction for such systems. Following their recommendations, our multistate XMCQDPT2 calculations used a $(12,12)$ reference space to provide a balanced description of the excited states. A series of XMCQDPT2 calculations with different denominator level shift values were performed to identify any intruder state influences, and allowed selection of the final value of 0.01 Hartree. No wavefunction symmetry restrictions were applied.

Collision cross-sections $\left(\Omega_{\mathrm{c}}\right)$ for all $E$ and $Z$ isomers of PABI and fragment ions were calculated using MOBCAL with the trajectory method parametrised for $\mathrm{N}_{2}$ buffer gas. ${ }^{48-50}$ Input charge distributions were computed at the $\omega \mathrm{B} 97 \mathrm{X}-\mathrm{D} / / \mathrm{cc}-\mathrm{pVTZ}$ level of theory with the Merz-Singh-Kollman scheme constrained to reproduce the electric dipole moment. ${ }^{51}$ Sufficient trajectories were computed to give calculated standard deviations less than $1 \AA^{2}$.

\section{Results and discussion}

\subsection{ATDs and isomer assignments}

The ion mobility data clearly show that PABI has two stable isomers in the gas phase with relative abundances that depend on the treatment of the ions. As shown in Fig. 3, ATDs for PABI recorded with low and high drive voltage for IF1 exhibit two peaks, whose relative intensities depend on the IF1 drive voltage. At high IF1 RF drive voltage violent collisions convert the faster isomer to the slower isomer prior to their injection into the IMS drift region suggesting that the slower isomer is the more stable form.

Calculated structures for the lowest energy $E$ and $Z$ isomers of PABI are shown in Fig. 1, with relative energies given in Table 1. Although the three bonds linking the two arene groups can each have $E$ or $Z$ configuration giving a total of eight isomers, only seven of these isomers are potential energy minima (see ESI $\dagger$ for more details). According to the calculations, two of the higher energy $E$ isomers ( $E 3$ and $E 4$ ) should thermally convert over relatively low barriers to the global $E$-isomer (E1). A second $E$-isomer (E2) is predicted to have a similar $\Omega_{\mathrm{c}}$ to $E 1$ and may also be present in small amounts. The $E 1$ and $E 2$ isomers are calculated to have similar excited states energies and are possibly indistinguishable in our experiment. The Z1-isomer of PABI (Fig. 1) is the only Z-isomer expected to be

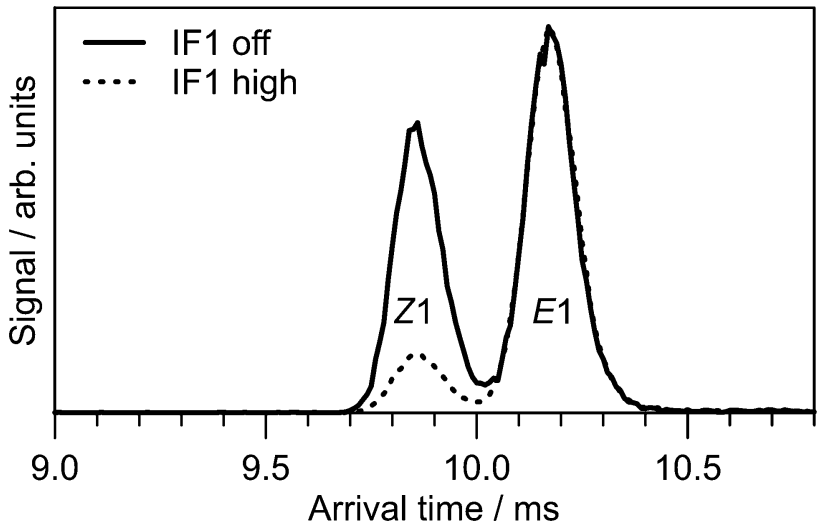

Fig. 3 ATDs for PABI with no RF drive voltage applied to IF1 (solid line) and high RF drive voltage applied to IF1 (dashed line). The two peaks are assigned to predominately the $E 1$ and $Z 1$ isomers.

Table 1 Calculated $\mathrm{PABI}$ isomer energies, $\triangle E$ (relative to the $E 1$-isomer), at the $\operatorname{CCSD}(\mathrm{T}) / \mathrm{cc}-\mathrm{pVTZ} / \mathrm{MP} 2 / \mathrm{cc}-\mathrm{pVTZ}$ level of theory, and collision cross-sections, $\Omega_{\mathrm{m}}$ (measured, $\pm 0.5 \AA^{2}$ ), $\Omega_{\mathrm{c}}$ (calculated)

\begin{tabular}{llll}
\hline Species & $\Delta E / \mathrm{kJ} \mathrm{mol}^{-1}$ & $\Omega_{\mathrm{m}} / \AA^{2}$ & $\Omega_{\mathrm{c}} / \AA^{2}$ \\
\hline$E 1$ & 0 & 139.8 & 146 \\
$Z 1$ & 5 & 135.2 & 141 \\
E-Azonium & 67 & - & 145 \\
$Z$-Azonium & 99 & - & 142 \\
\hline
\end{tabular}

present in our experiments based on its relative stability and low isomerisation barriers for conversion to other $Z$-isomers.

The $\operatorname{CCSD}(\mathrm{T})$ benchmark calculations (Table 1) predict the E1-isomer to be $5 \mathrm{~kJ} \mathrm{~mol}^{-1}$ more stable than the Z1-isomer, suggesting the slower peak in Fig. 3 is associated with the E1-isomer. This assignment is consistent with calculated $\left(\Omega_{\mathrm{c}}\right)$ and measured $\left(\Omega_{\mathrm{m}}\right)$ collision cross-sections, which are included in Table $1 . \Omega_{\mathrm{c}}$ values for other $E$ and $Z$ isomers are given in the ESI. $\dagger$ The $\approx 4 \%$ overestimation of $\Omega_{\mathrm{c}}$ compared with $\Omega_{\mathrm{m}}$ is consistent with measurements and calculations for other azo cations. ${ }^{52}$

It is worth noting that the minimum energy $E$-azonium and $Z$-azonium protomers of PABI, in which a proton is attached to an $\mathrm{N}$ atom of the azo group, are calculated to lie much higher in energy E1-PABI (Table 1) and are unlikely to be important in our experiment.

\subsection{Photoisomerisation of $E 1$ and Z1-PABI}

Exposing $E$ and $Z$ PABI to light in the drift region caused photoisomerisation. For example, Fig. 4a shows light-off, lighton, and difference (light-on-light-off) ATDs following exposure of the $E 1$-isomer to $460 \mathrm{~nm}$ light. The difference ATD clearly shows depletion of the $E 1$-isomer and its photoconversion to the Z1-isomer. $\$$ The photoisomerisation efficiency depends on

$\ddagger$ Because the ions were irradiated approximately halfway along the drift region (after IMS1 and IG2), the photoisomer peak ( $Z 1$ for $E-Z$ photoconversion or $E 1$ for $Z-E$ photoconversion) appears between the parent isomer peak and the peak for the product isomer when it is separated over both drift regions (IMS1 + IMS2). The same situation prevails for the slammer measurements described in Section 4.4. Note that in all presented ATDs $t=0$ corresponds to the opening of IG1. 

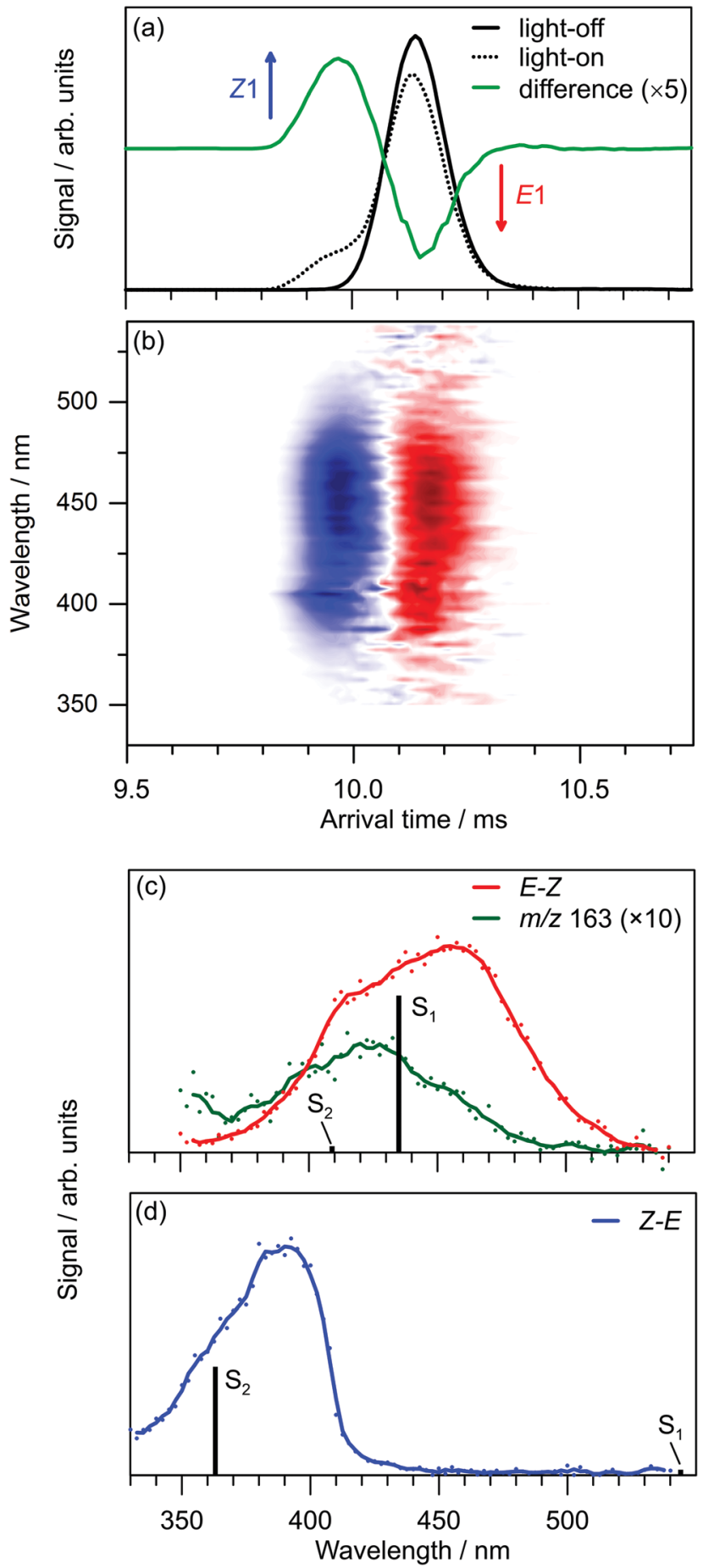

Fig. 4 Photoisomerisation of PABI: (a) light-off, light-on, and difference ATDs with $460 \mathrm{~nm}$ irradiation of the $E 1$-isomer; (b) $E 1$ (red - depletion) to Z1 (blue - growth) photoisomerisation action signal for wavelengths between 350-575 nm; (c) E-Z PISA spectrum; and (d) Z-E PISA spectrum. Included in (c) is the $\mathrm{m} / \mathrm{z} 163$ photofragment curve. The calculated $E 1\left(\mathrm{~S}_{1}\right), E 1\left(\mathrm{~S}_{2}\right), Z 1\left(\mathrm{~S}_{1}\right)$ and $Z 1\left(S_{2}\right)$ state energies of PABI (vertical bars in (c and d)) were determined at the multistate XMCQDPT2/GEN/MP2/cc-pVTZ level of theory.

wavelength, as shown Fig. 4b, which displays the difference ATD as a function of OPO wavelength. Integrating over the $Z 1$-isomer appearance peak in Fig. $4 \mathrm{~b}$ yields the $E-Z$ photoisomerisation action (PISA) spectrum shown in Fig. 4c, which exhibits a broad photoisomerisation response over the 540-350 nm range, peaking at $460 \mathrm{~nm}(2.70 \mathrm{eV})$. The peak in the corresponding $Z-E$ PISA spectrum, which is shown in Fig. 4d, has a maximum at $390 \mathrm{~nm}$ $(3.18 \mathrm{eV})$, blue-shifted by $\approx 70 \mathrm{~nm}(0.48 \mathrm{eV})$ relative to the peak in the $E-Z$ PISA spectrum. A similar difference between the $E 1$ and $Z 1$ isomer absorption is predicted by high level multistate XMCQDPT2 calculations, summarised in Fig. 5. These calculations predict the bright $\mathrm{S}_{1}$ state of the $E 1$-isomer has $\pi \pi^{*}$ character and occurs at $435 \mathrm{~nm}(2.85 \mathrm{eV}$, oscillator strength 1.0), whereas the optically dark $S_{2}$ state with $n \pi^{*}$ character occurs at $413 \mathrm{~nm}$ (oscillator strength 0.02 ). This $S_{1}$ and $S_{2}$ state ordering is reversed compared with $E$-azobenzene because the $\mathrm{n}$ orbital in PABI is stabilised by the added proton thereby increasing the energy of the $n \pi^{*}$ excitation. For the $Z 1$-isomer the optically dark $n \pi^{*} S_{1}$ state occurs at $540 \mathrm{~nm}$ (oscillator strength 0.01), while the bright $S_{2} \pi \pi^{*}$ state occurs at $363 \mathrm{~nm}$ (3.42 eV, oscillator strength 0.84). Thus, the XMCQDPT2 calculations are consistent with the PISA spectra in predicting that the $Z 1$-isomer absorbs $\approx 0.5 \mathrm{eV}$ higher in energy than the E1-isomer. The results of EOM-CCSD calculations are also broadly consistent with the multistate XMCQDPT2 calculations and give a similar separation for the bright states of the $E 1$ and $Z 1$-isomers.

The marked difference between the PISA spectra of $E 1$ and $Z 1$ PABI isomers is at odds with the results for PABI in methanol solution reported by Weston et al. ${ }^{17}$ who deduced that $E$ and $Z$ PABI had similar absorption spectra, a result that was supported by accompanying TD-DFT calculations [CAMB3LYP//6-311G(2df,2p) level] with a PCM continuum model. The UV-vis absorption spectrum of PABI in methanol at low$\mathrm{pH}$ reported by Weston et al., ${ }^{17}$ which appears to have a maximum at $\approx 440 \mathrm{~nm}$ is similar to the $E-Z$ PISA spectrum. Comparisons between solution and gas-phase spectra for the $Z$-isomer are more difficult. Weston et $a{ }^{17}{ }^{17}$ reported an absorption spectrum for the photostationary state (PSS) established at $415 \mathrm{~nm}$, which contains a mixture of $E$ and $Z$-isomers (and perhaps also isomers of the neutral ABI molecule which absorb in the same range). The absorption spectrum of the PSS is similar to that of the $E$-isomer with a minor diminution of the main peak intensity, an observation that is consistent with overlapping $E$-isomer and $Z$-isomer bands for PABI in methanol.§ Reconciling the solution and gas-phase spectra of the $E$ and $Z$ isomers would require that methanol solvation of the $Z$ isomer leads to a substantial red shift $(\approx 50 \mathrm{~nm})$ in its absorption maximum.

\subsection{Photodissociation of E1-PABI}

A small fraction of PABI photodissociated at wavelengths shorter than $470 \mathrm{~nm}$ to yield a $m / z \quad 163$ photoproduct,

$\S$ Using IMS, we confirmed that PSSs established at $473 \mathrm{~nm}$ and $438 \mathrm{~nm}$ contain an appreciable fraction of the $Z$-isomer $(\approx 60 \% Z$-isomer at $473 \mathrm{~nm}, \approx 40 \%$ $Z$-isomer at $438 \mathrm{~nm}$ ). Note that $Z-E$ thermal reversion from the PSS at $\mathrm{pH} 4$ is slow $\left(t_{1 / 2}=1860 \mathrm{~s}\right.$, see ESI $\left.\dagger\right)$ compared with the sampling time of our experiment $(\approx 60 \mathrm{~s})$, so measured ATDs should reflect PSS populations. ${ }^{52}$ 
$E_{1}: S_{1}$

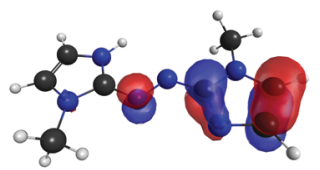

п [HOMO]

$E 1: S_{2}$

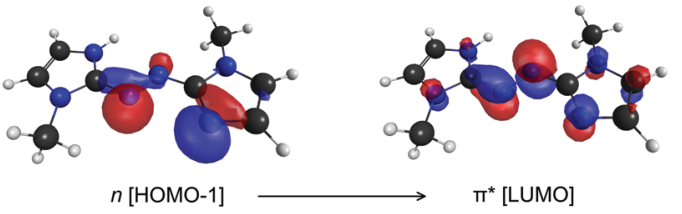

Z1: $S_{1}$

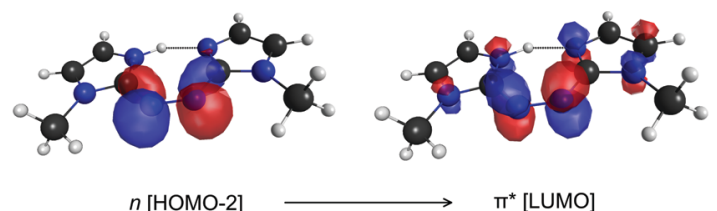

$Z 1: S_{2}$

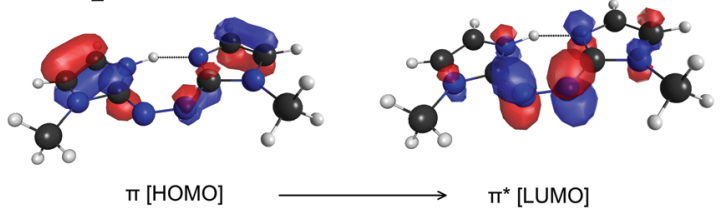

Fig. 5 Predominant orbital excitations (CASSCF natural orbitals) to give the $S_{1}$ and $S_{2}$ states of E1-PABI and Z1-PABI.

consistent with loss of a $\mathrm{N}_{2}$ molecule. The $m / z 163$ degradation product could also be formed by exposing the PABI solution in the syringe with continuous $473 \mathrm{~nm}$ irradiation for several hours. $\mathrm{N}_{2}$ loss from azo and diazo compounds is a well-known thermal and photochemical decomposition process in both the gas phase and in solution. ${ }^{53-55}$ There is evidence for two $\mathrm{m} / \mathrm{z} 163$ isomers with the ATD for the $m / z 163$ photofragments (Fig. 6) displaying two peaks. The slower, more intense peak is assigned to protonated

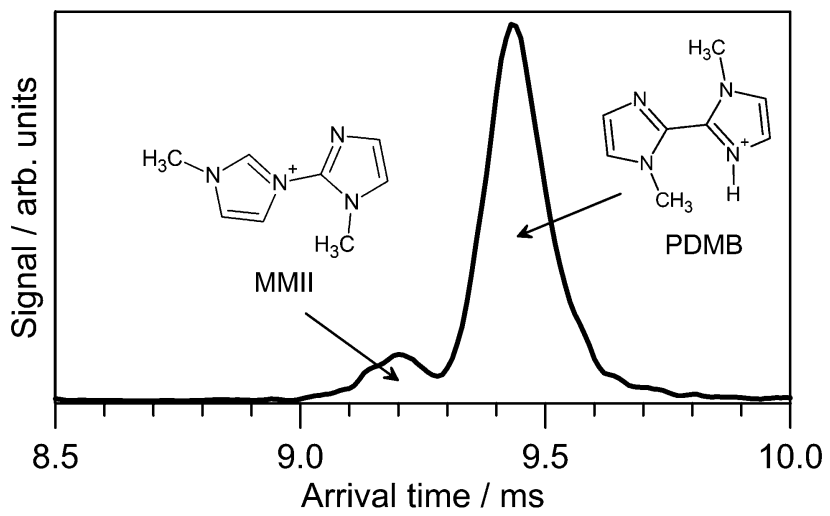

Fig. 6 ATD recorded at $m / z 163$ following $473 \mathrm{~nm}$ irradiation of E1-PABI. Proposed PDMB and MMII isomer structures are indicated. $1,1^{\prime}$-dimethyl-biimidazole (PDMB) whereas the faster peak is assigned to 1-methyl-3-(1-methyl-imidazol-2-yl)-1 $H$-imidazol3-ium (MMII) which is calculated (DLPNO-CCSD(T)/cc-pVTZ/ $\omega B 97 X D / c c-p V T Z$ level) to lie $42 \mathrm{~kJ} \mathrm{~mol}^{-1}$ higher in energy. The isomer in which a ring tertiary nitrogen atom is protonated was calculated to lie $>100 \mathrm{~kJ} \mathrm{~mol}^{-1}$ higher in energy than $E$-PDMB.

The photodissociation action spectrum of PABI, obtained by monitoring the $m / z 163$ fragment (Fig. 4a), is blue-shifted compared to the PISA spectrum with the peak maxima separated by $\approx 50 \mathrm{~nm}$. Although the reason for the blue shift is unclear, it may be associated with an energy barrier for the $\mathrm{N}_{2}$ loss channel, such that the process is more efficient for higher energy photons. CCSD(T)/cc-pVTZ/ $\omega$ B97X-D/cc-pVTZ calculations determined the $E 1$-PABI $\rightarrow E$-PDBM $+\mathrm{N}_{2}$ channel to be exoergic with $\Delta E=-27 \mathrm{~kJ} \mathrm{~mol}^{-1}$ (the $\omega \mathrm{B} 97 \mathrm{X}-\mathrm{D} / / \mathrm{cc}-\mathrm{pVTZ}$ value is $-8 \mathrm{~kJ} \mathrm{~mol}^{-1}$ ), suggesting that PABI should be susceptible to thermal decomposition in the gas-phase. In separate collisional excitation experiments, we confirmed that $\mathrm{N}_{2}$ loss is also the predominant collision-induced dissociation channel and that it has an onset somewhat above that for $E-Z$ isomerisation (Section 4.4).

\subsection{Collision-induced isomerisation of $E 1$ and Z1-PABI}

The transformations of PABI on the ground state potential energy surface were investigated by colliding the ions with $\mathrm{N}_{2}$ buffer gas in the slammer region. As an example, Fig. 7a illustrates the effect of collisions on the $Z 1$-isomer over a range of slammer potential differences $(\Delta V)$. There is an obvious conversion of the Z1-isomer to the E1-isomer with an onset over the 90-130 $\mathrm{V}$ range. The presence of both isomers in this range is apparent in an ATD recorded with $\Delta V=100 \mathrm{~V}$ (Fig. 7b). One can construct breakdown diagrams by fitting ATDs for each $\Delta V$ to a sum of $Z 1$ and $E 1$ Gaussian curves and plotting the amplitudes against $\Delta V$. Breakdown curves, obtained starting with mobility-selected $Z 1$ and E1-isomers, are shown in Fig. 7c and $\mathrm{d}$, respectively. Analysis of the curves shows that $E-Z$ isomerisation has a $88 \pm 3 \mathrm{~V}$ threshold (Fig. 7c), whereas $Z-E$ isomerisation has a $75 \pm 3 \mathrm{~V}$ threshold (Fig. 7d). The ordering of these thresholds is consistent with the greater stability of the E1-isomer. As can be seen from Fig. 7c and d, a quasiequilibrium is established over the $\Delta V=130-180 \mathrm{~V}$ range with a $0.12: 0.88 Z 1: E 1$ population ratio, independent of the initially selected isomer, again consistent with the greater stability of the $E 1$-isomer.

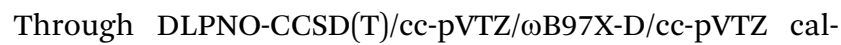
culations, we identified two ground state isomerisation pathways, illustrated in Fig. 8, corresponding to an inversion mechanism (barrier of $133 \mathrm{~kJ} \mathrm{~mol}^{-1}$ ) and a torsion mechanism (barrier of $137 \mathrm{~kJ} \mathrm{~mol}^{-1}$ ). Because the two pathways have similar barriers, both should contribute to collision-induced isomerisation in the gas phase. Note that $Z-E$ isomerisation through the inversion mechanism first forms $E 2$, which then rotates about a single bond to form the E1-isomer. The predicted importance of both the inversion and torsion pathways for PABI differs from the situation for neutral 

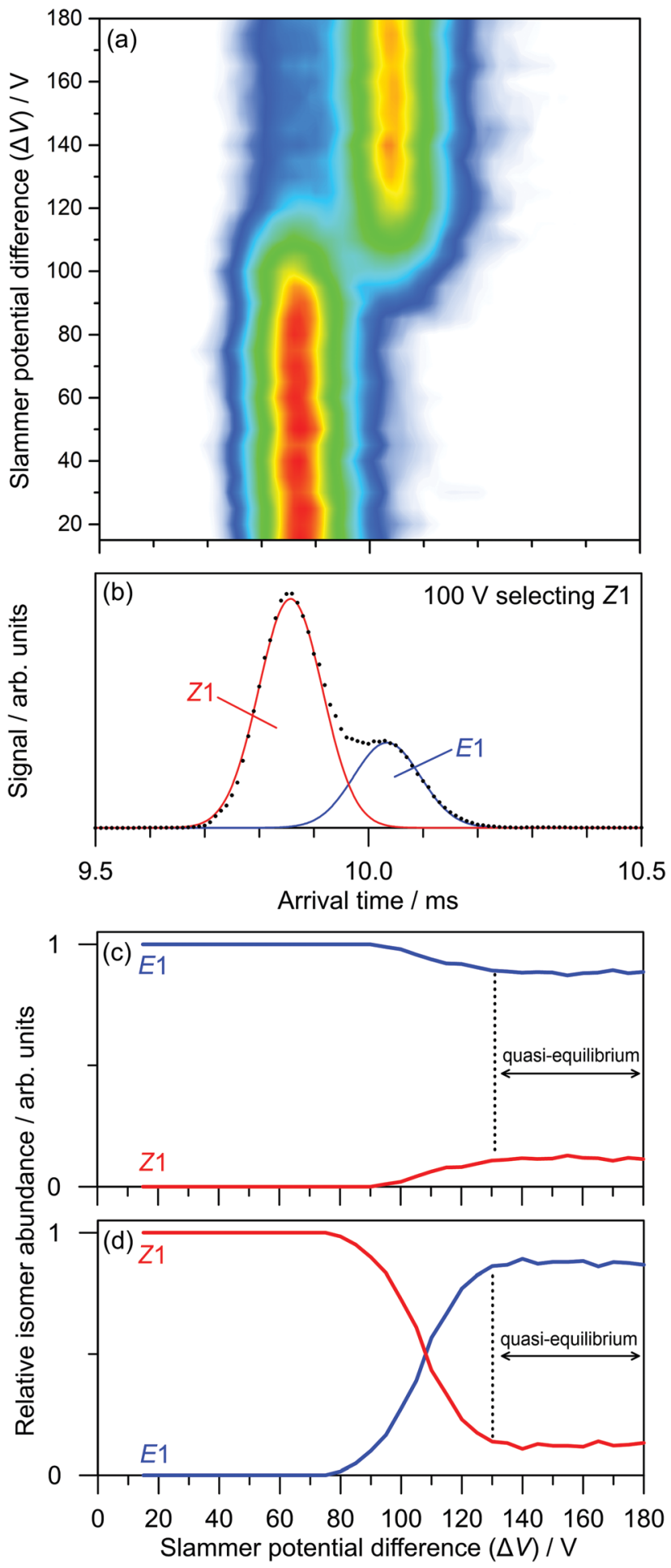

Fig. 7 Collision-induced isomerisation of PABI: (a) ATDs as a function of slammer potential difference $(\Delta V$ ) for initial mobility-selected Z1-PABI; (b) example $\Delta V=100 \mathrm{~V}$ ATD fit to extract abundances of the $E 1$ and $Z 1$ isomers; (c) and (d) isomer abundances as a function of $\Delta V$ following initial mobility-selection of $E 1$ and $Z 1$ isomers, respectively.

azobenzene, for which it is well established that thermal isomerisation proceeds through an inversion mechanism with a barrier of $189 \mathrm{~kJ} \mathrm{~mol}^{-1},{ }^{24}$ somewhat larger than for PABI.

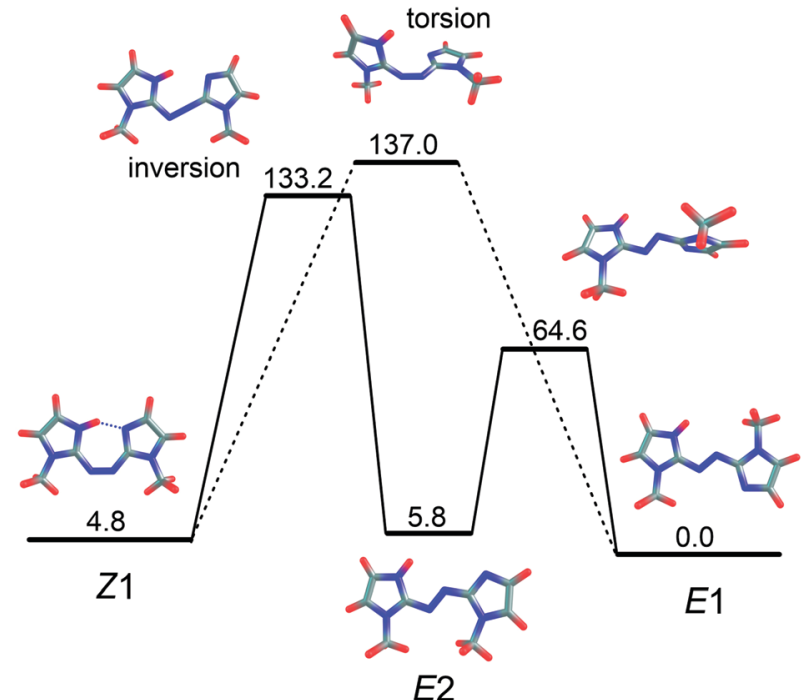

Fig. $8 Z-E$ collision-induced isomerisation pathways of $P A B I$ calculated

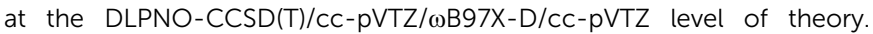
Energies are given in $\mathrm{kJ} \mathrm{mol}^{-1}$ relative to $E 1$. Key: solid lines - inversion mechanism; dashed lines - torsion mechanism.

\section{Conclusions}

We have characterised the photoisomerisation of protonated azobis(2-imidazole), an intramolecular hydrogen-bonded photoswitch. In the gas phase, $E-Z$ photoisomerisation occurs over the $360-520 \mathrm{~nm}$ range, and $Z-E$ photoisomerisation occurs over the $420-320 \mathrm{~nm}$ range. The peak in the gas-phase photoisomerisation spectrum of the $Z$-isomer is blue-shifted by $\approx 70 \mathrm{~nm}$ relative to the $E$-isomer (consistent with the predictions from XMCQDPT2 calculations) implying that in the gas phase PABI fulfils one of the primary requirements of an ideal molecular switch - reversible photoswitching with different colours of light. Loss of an $\mathrm{N}_{2}$ molecule is identified as the predominant photo and collisional decomposition pathway.

\section{Acknowledgements}

This research was funded through the Australian Research Council Discovery Project scheme (DP150101427 and DP160100474). Computational resources were provided by the Australian National Computational Infrastructure (NCI) through award of Early Career Allocation ya1 to JNB.

\section{References}

1 J. Calbo, C. E. Weston, A. J. P. White, H. S. Rzepa, J. Contreras-Garcia and M. J. Fuchter, J. Am. Chem. Soc., 2017, 139, 1261.

2 A. A. Beharry and G. A. Woolley, Chem. Soc. Rev., 2011, 40, 4422.

3 H. M. D. Bandara and S. C. Burdette, Chem. Soc. Rev., 2012, 41, 1809. 
4 A. A. Beharry, L. Wong, V. Tropepe and G. A. Woolley, Angew. Chem., Int. Ed., 2011, 50, 1325.

5 T. Hugel, N. B. Holland, A. Cattani, L. Moroder, M. Seitz and H. E. Gaub, Science, 2002, 296, 1103.

6 T. Muraoka, K. Kinbara and T. Aida, Nature, 2006, 440, 512.

7 W. R. Browne and B. L. Feringa, Nat. Nanotechnol., 2006, 1, 25.

8 Z. F. Liu, K. Hashimoto and A. Fujishima, Nature, 1990, 347, 658.

9 A. Ida, B. Cohen, T. Asaka, A. Kawai, J. A. Organero, K. Shibuya and A. Douhal, Phys. Chem. Chem. Phys., 2011, 13, 20318.

10 P. Hamm, J. Helbing and J. Bredenbeck, Annu. Rev. Phys. Chem., 2008, 59, 291.

11 C. Renner and L. Moroder, ChemBioChem, 2006, 7, 869.

12 W. Szymanński, J. M. Beierle, H. A. V. Kistemaker, W. A. Velema and B. L. Feringa, Chem. Rev., 2013, 113, 6114.

13 Y. Kim, J. A. Phillips, H. Liu, H. Kang and W. Tan, Proc. Natl. Acad. Sci. U. S. A., 2009, 106, 6489.

14 W. A. Velema, W. Szymanski and B. L. Feringa, J. Am. Chem. Soc., 2014, 136, 2178.

15 M. Borowiak, W. Nahaboo, M. Reynders, K. Nekolla, P. Jalinot, J. Hasserodt, M. Rehberg, M. Delattre, S. Zahler, A. Vollmar, D. Trauner and O. Thorn-Seshold, Cell, 2015, $162,403$.

16 M. R. Banghart, A. Mourot, D. L. Fortin, J. Z. Yao, R. H. Kramer and D. Trauner, Angew. Chem., Int. Ed., 2009, 48, 9097.

17 C. E. Weston, R. D. Richardson and M. J. Fuchter, Chem. Commun., 2016, 52, 4521.

18 J. Otsuki, K. Suwa, K. K. Sarker and C. Sinha, J. Phys. Chem. A, 2007, 111, 1403.

19 T. Schultz, J. Quenneville, B. Levine, A. Toniolo, T. J. Martínez, S. Lochbrunner, M. Schmitt, J. P. Shaffer, M. Z. Zgierski and A. Stolow, J. Am. Chem. Soc., 2003, 125, 8098.

20 E. M. M. Tan, S. Amirjalayer, S. Smolarek, A. Vdovin, F. Zerbetto and W. J. Buma, Nat. Commun., 2015, 6, 5860.

21 T. Fujino, S. Y. Arzhantsev and T. Tahara, J. Phys. Chem. A, 2001, 105, 8123.

22 T. Cusati, G. Granucci and M. Persico, J. Am. Chem. Soc., 2011, 133, 5109.

23 M. Quick, A. L. Dobryakov, M. Gerecke, C. Richter, F. Brendt, I. N. Ioffe, A. A. Granovsky, R. Mahrwald, N. P. Ernsting and S. A. Kovalenko, J. Phys. Chem. B, 2014, 118, 8756.

24 J. Casellas, M. J. Bearpark and M. Reguero, ChemPhysChem, 2016, 17, 1.

25 I. Conti, M. Garavelli and G. Orlandi, J. Am. Chem. Soc., 2008, 130, 5216.

26 B. D. Adamson, N. J. A. Coughlan, R. E. Continetti and E. J. Bieske, Phys. Chem. Chem. Phys., 2013, 15, 9540.

27 B. D. Adamson, N. J. A. Coughlan, G. da Silva and E. J. Bieske, J. Phys. Chem. A, 2013, 117, 13319.

28 N. J. A. Coughlan, B. D. Adamson, K. J. Catani, U. Wille and E. J. Bieske, J. Phys. Chem. Lett., 2014, 5, 3195.

29 N. J. A. Coughlan, K. J. Catani, B. D. Adamson, U. Wille and E. J. Bieske, J. Chem. Phys., 2014, 140, 164307.
30 N. J. A. Coughlan, B. D. Adamson, L. Gamon, K. Catani and E. J. Bieske, Phys. Chem. Chem. Phys., 2015, 17, 22623.

31 P. B. Markworth, N. J. A. Coughlan, B. D. Adamson, L. Goerigk and E. J. Bieske, Phys. Chem. Chem. Phys., 2015, 17, 25676.

32 I. Czerwinska, A. Kulesza, C. Choi, F. Chirot, A.-L. Simon, J. Far, C. Kune, E. de Pauw and P. Dugourd, Phys. Chem. Chem. Phys., 2016, 18, 32331.

33 B. D. Adamson, N. J. A. Coughlan, P. B. Markworth, R. E. Continetti and E. J. Bieske, Rev. Sci. Instrum., 2014, 85, 123109.

34 N. A. Pierson, S. J. Valentine and D. E. Clemmer, J. Phys. Chem. B, 2010, 114, 7777.

35 N. A. Pierson and D. E. Clemmer, Int. J. Mass Spectrom., 2015, 377, 646.

36 J. M. Turney, A. C. Simmonett, R. M. Parrish, E. G. Hohenstein, F. Evangelista, J. T. Fermann, B. J. Mintz, L. A. Burns, J. J. Wilke, M. L. Abrams, N. J. Russ, M. L. Leininger, C. L. Janssen, E. T. Seidl, W. D. Allen, H. F. Schaefer, R. A. King, E. F. Valeev, C. D. Sherrill and T. D. Crawford, Wiley Interdiscip. Rev.: Comput. Mol. Sci., 2012, 2, 556.

37 M. W. Schmidt, K. K. Baldridge, J. A. Boatz, S. T. Elbert, M. S. Gordon, J. H. Jensen, S. Koseki, N. Matsunaga, K. A. Nguyen, S. Su, T. L. Windus, M. Dupuis and J. A. Montgomery, J. Comput. Chem., 1993, 14, 1347.

38 F. Neese, Wiley Interdiscip. Rev.: Comput. Mol. Sci., 2012, 2, 73. 39 M. J. Frisch, G. W. Trucks, H. B. Schlegel, G. E. Scuseria, M. A. Robb, J. R. Cheeseman, G. Scalmani, V. Barone, B. Mennucci, G. A. Petersson, H. Nakatsuji, M. Caricato, X. Li, H. P. Hratchian, A. F. Izmaylov, J. Bloino, G. Zheng, J. L. Sonnenberg, M. Hada, M. Ehara, K. Toyota, R. Fukuda, J. Hasegawa, M. Ishida, T. Nakajima, Y. Honda, O. Kitao, H. Nakai, T. Vreven, J. A. Montgomery, Jr., J. E. Peralta, F. Ogliaro, M. Bearpark, J. J. Heyd, E. Brothers, K. N. Kudin, V. N. Staroverov, R. Kobayashi, J. Normand, K. Raghavachari, A. Rendell, J. C. Burant, S. S. Iyengar, J. Tomasi, M. Cossi, N. Rega, J. M. Millam, M. Klene, J. E. Knox, J. B. Cross, V. Bakken, C. Adamo, J. Jaramillo, R. Gomperts, R. E. Stratmann, O. Yazyev, A. J. Austin, R. Cammi, C. Pomelli, J. W. Ochterski, R. L. Martin, K. Morokuma, V. G. Zakrzewski, G. A. Voth, P. Salvador, J. J. Dannenberg, S. Dapprich, A. D. Daniels, Ö. Farkas, J. B. Foresman, J. V. Ortiz, J. Cioslowski and D. J. Fox, Gaussian 09 Revision D.01, Gaussian Inc., Wallingford, CT, 2009.

40 J. A. Pople, M. Head-Gordon and K. Raghavachari, J. Chem. Phys., 1987, 87, 5968.

41 C. Riplinger, B. Sandhoefer, A. Hansen and F. Neese, J. Chem. Phys., 2013, 139, 134101.

42 M. J. Frisch, M. Head-Gordon and J. A. Pople, Chem. Phys. Lett., 1990, 166, 281.

43 J.-D. Chai and M. Head-Gordon, Phys. Chem. Chem. Phys., 2008, 10, 6615.

44 T. H. Dunning, Jr., J. Chem. Phys., 1989, 90, 1007. 45 A. A. Granovsky, J. Chem. Phys., 2011, 134, 214113. 
46 J. F. Stanton and R. J. Bartlett, J. Chem. Phys., 1993, 98, 7029. 47 I. N. Ioffe and A. A. Granovsky, J. Chem. Theory Comput., 2013, 9, 4973.

48 A. A. Shvartsburg and M. F. Jarrold, Chem. Phys. Lett., 1996, 261, 86.

49 M. Mesleh, J. Hunter, A. Shvartsburg, G. Schatz and M. Jarrold, J. Phys. Chem., 1996, 100, 16082.

50 I. Campuzano, M. F. Bush, C. V. Robinson, C. Beaumont, K. Richardson, H. Kim and H. I. Kim, Anal. Chem., 2012, 84, 1026.
51 B. H. Besler, K. M. Merz, Jr. and P. A. Kollman, J. Comput. Chem., 1990, 11, 431.

52 J. N. Bull, M. S. Scholz, N. J. A. Coughlan, A. Kawai and E. J. Bieske, Anal. Chem., 2016, 88, 11978.

53 P. S. Engel, Chem. Rev., 1980, 80, 99.

54 D. Srzic, M. Žinić, Z. Meić, G. Czira and J. Tainás, Org. Mass Spectrom., 1992, 27, 1305.

55 A. Leiba and I. Oref, J. Chem. Soc., Faraday Trans. 1, 1979, 75, 2694. 